\title{
High-resolution contrast-enhanced optical coherence tomography in mice retinae
}

\author{
Debasish Sen \\ Elliott D. SoRelle \\ Orly Liba \\ Roopa Dalal \\ Yannis M. Paulus \\ Tae-Wan Kim \\ Darius M. Moshfeghi \\ Adam de la Zerda
}




\title{
High-resolution contrast-enhanced optical coherence tomography in mice retinae
}

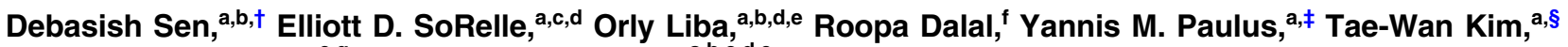 \\ Darius M. Moshfeghi, ${ }^{e, g}$ and Adam de la Zerda ${ }^{a, b, c, d, e, *}$ \\ a Stanford University, Department of Structural Biology, 299 Campus Drive, Stanford, California 94305, United States \\ bStanford University, Molecular Imaging Program at Stanford, 299 Campus Drive, Stanford, California 94305, United States \\ 'Stanford University, Biophysics Program, 299 Campus Drive, Stanford, California 94305, United States \\ dStanford University, Department of Electrical Engineering, 299 Campus Drive, Stanford, California 94305, United States \\ eStanford University, Bio-X Program, 299 Campus Drive, Stanford, California, 94305, United States \\ 'Stanford University, Department of Ophthalmology, 300 Pasteur Drive, Palo Alto, California 94304, United States \\ 9Stanford University, Department of Ophthalmology, Stanford Byers Eye Institute, 2452 Watson Court, Palo Alto, California 94303, United States
}

\begin{abstract}
Optical coherence tomography (OCT) is a noninvasive interferometric imaging modality providing anatomical information at depths of millimeters and a resolution of micrometers. Conventional OCT images limit our knowledge to anatomical structures alone, without any contrast enhancement. Therefore, here we have, for the first time, optimized an OCT-based contrast-enhanced imaging system for imaging single cells and blood vessels in vivo inside the living mouse retina at subnanomolar sensitivity. We used bioconjugated gold nanorods (GNRs) as exogenous OCT contrast agents. Specifically, we used anti-mouse CD45 coated GNRs to label mouse leukocytes and mPEG-coated GNRs to determine sensitivity of GNR detection in vivo inside mice retinae. We corroborated OCT observations with hyperspectral dark-field microscopy of formalin-fixed histological sections. Our results show that mouse leukocytes that otherwise do not produce OCT contrast can be labeled with GNRs leading to significant OCT intensity equivalent to a $0.5 \mathrm{nM}$ GNR solution. Furthermore, GNRs injected intravenously can be detected inside retinal blood vessels at a sensitivity of $\sim 0.5 \mathrm{nM}$, and GNR-labeled cells injected intravenously can be detected inside retinal capillaries by enhanced OCT contrast. We envision the unprecedented resolution and sensitivity of functionalized GNRs coupled with OCT to be adopted for longitudinal studies of retinal disorders. ๑ 2016 Society of Photo-Optical Instrumentation Engineers (SPIE) [DOI: 10.1117/1.JBO.21.6.066002]
\end{abstract}

Keywords: optical coherence tomography; gold nanorods; retina; in vivo imaging; leukocyte.

Paper 150578RR received Aug. 28, 2015; accepted for publication May 2, 2016; published online Jun. 6, 2016.

\section{Introduction}

Optical coherence tomography (OCT) is a noninvasive imaging modality that uses low-coherence interferometry to detect optical scattering from tissue, cells, or objects with lateral spatial resolutions of a few microns up to several millimeters deep into tissue. ${ }^{1,2}$ Thus, a noninvasive technology like OCT can be very useful for longitudinal studies of the cellular and molecular foundations of diseased conditions. Because of the lack of an OCT based imaging system that is capable of providing information via functionalized contrast agents, our knowledge is currently limited to visual clues obtained with conventional OCT images of anatomical structures and/or anomalies within tissues. However, these anomalies are merely the outcome of complex molecular and cellular interactions. Without the benefit of enhanced molecular contrast in OCT imaging, it is difficult to determine the role of the aforementioned complex structural anomalies in a diseased condition. Here we seek to optimize an OCT-based imaging technique coupled with functionalized

\footnotetext{
*Address all correspondence to: Adam de la Zerda, E-mail: adlz@ stanford.edu

${ }^{\dagger}$ Current address: Asterias Biotherapeutics, Fremont, California 94555, United States

${ }^{\ddagger}$ Current address: University of Michigan, Kellogg Eye Center, Grand Blanc, Michigan 48439, United States

${ }^{\S}$ Current address: Seoul Metropolitan Government, Seoul National University Boramae Medical Center, Department of Ophthalmology, Seoul, Republic of
} Korea contrast agents as a multifaceted technology for imaging single cells and blood vessels in vivo inside the mouse retina noninvasively at subnanomolar sensitivity.

Following our development of numerous imaging agents for specifically labeling target proteins of interest in living mice, ${ }^{3-6}$ we recently designed a new OCT-based imaging system for the retina by developing nanoparticles based on gold nanorods (GNRs), which produce a strong OCT signal. GNRs have been recently used to enhance OCT signals in tissue mimicking phantoms, ${ }^{7-11}$ excised porcine eyes, ${ }^{8}$ vitreous of mice eyes, ${ }^{12}$ anterior chambers and cornea of mice eyes, ${ }^{13}$ and in mouse mammary tumors. ${ }^{14}$ Additionally, using our custom synthesized GNRs $(\sim 100 \times 30 \mathrm{~nm})$, we have achieved ultrahigh detection sensitivity in vivo. ${ }^{15}$ Here we sought to address whether freshly isolated leukocytes could be labeled with GNRs to enhance scattering, and thus be detected in vitro and in vivo in the retina using OCT. We further determined the sensitivity for detection of GNRs inside retinal blood vessels using OCT in vivo. To our knowledge, this is a first demonstration of OCT-based cellular imaging at a high resolution and subnanomolar sensitivity, allowing single cell and blood capillary detection inside the retina in vivo.

OCT has long been used to image human eyes for clinical diagnosis $^{1,2,16}$ and several commercial OCT systems are FDA approved for ophthalmological use. GNRs were previously

1083-3668/2016/\$25.00 (c) 2016 SPIE 
shown to be nontoxic ${ }^{13,17}$ and have also been used for clinical trials in cancer immunotherapy ${ }^{18}$ and photothermal cancer therapy. ${ }^{19,20}$ So, we expect our OCT imaging system paired with a nonsurgical minimally invasive procedure to be fully translatable to patients in the future. Additionally, depth and resolution offered by OCT, as well as the enhanced contrast of functionalized GNRs may complement existing clinical imaging techniques and help in better diagnosis and personalized treatments of disorders.

\section{Methods}

\subsection{Functionalized Gold Nanorods}

GNRs stable in aqueous solutions were synthesized as previously reported. ${ }^{15}$ These GNRs $(\sim 110 \times 32 \mathrm{~nm})$ exhibited peak longitudinal surface plasmon resonance between 824 and $830 \mathrm{~nm}$, and a spectral full width at half maximum (FWHM) of $100 \mathrm{~nm}$. GNR absorbance was verified using a UV-Vis spectrophotometer. After synthesis, GNRs were coated with poly(sodium 4-styrenesulfonate) (PSS), yielding GNRPSS conjugates. Using thiol reactivity, GNR-PSS was further conjugated with either mPEG or PEG-Biotin to make GNRmPEG conjugates $\left(\mathrm{GNR}^{\mathrm{mPEG}}\right)$ or GNR-biotin conjugates, respectively. $10 \mathrm{nM}$ GNR-biotin conjugates were incubated with NeutrAvidin at $2 \mu \mathrm{g} / \mathrm{mL}$ final concentration and washed twice at $2250 \times g$ at room temperature $\left(\mathrm{RT}, 25^{\circ} \mathrm{C}\right)$. The pellets of NeutrAvidin-conjugated GNRs $\left(\mathrm{GNR}^{\mathrm{Nav}}\right)$ were resuspended in distilled deionized water $\left(\mathrm{diH}_{2} \mathrm{O}\right)$ to a final concentration of $10 \mathrm{nM}$. Stability of $\mathrm{GNR}^{\mathrm{Nav}}$ was tested in various solutions as follows. $5 \mu \mathrm{L}$ of GNR ${ }^{\mathrm{Nav}}$ was added to $95 \mu \mathrm{L}$ of either $\mathrm{diH}_{2} \mathrm{O}$ or Dulbecco's modified Eagle's medium (DMEM, Gibco) alone, or $\mathrm{diH}_{2} \mathrm{O}$ or DMEM containing 5 or $10 \%$ fetal bovine serum (FBS, Hyclone) and optical density (OD) was measured immediately. The solutions containing $\mathrm{GNR}^{\mathrm{Nav}}$ were then incubated for $1 \mathrm{~h}$ at RT, centrifuged at $2250 \times g$ for $10 \mathrm{~min}$ at RT, resuspended without discarding the supernatant, and then OD was measured again. The ratio of the OD before and after incubation was used to determine GNR stability in these solutions.
We observed that GNR stability was significantly reduced in physiological solutions in the absence of FBS. Thus, for all experiments involving GNRs in physiological solutions, 5\% FBS was used.

\subsection{Labeling of Leukocytes with Gold Nanorod}

Eight-week-old nu/nu mice (Charles River) were euthanized using $\mathrm{CO}_{2}$, and spleens and blood were harvested. The spleen was homogenized to get single cell suspensions and mixed with the blood [Fig. 1(a)]. The cells were then washed twice in DMEM, and red blood cells (RBCs) were lysed using $1 \times$ lysis buffer (Biolegend) following manufacturer's protocol. $2.5 \mu \mathrm{g}$ of biotin-conjugated anti-mouse CD45 (Biolegend, Clone 30-F11) was incubated for $20 \mathrm{~min}$ at RT with $50 \mu \mathrm{L}$ of OD $200 \mathrm{GNR}^{\mathrm{Nav}}$ (see above) + diluted to $1 \mathrm{~mL}$ using $\mathrm{diH}_{2} \mathrm{O}$ to make GNR-anti-CD45 conjugates $\left(\mathrm{GNR}^{\mathrm{CD} 45}\right)$. GNR ${ }^{\mathrm{CD} 45}$ was washed twice in $\mathrm{diH}_{2} \mathrm{O}$ by centrifuging at $2250 \times g$ for $10 \mathrm{~min}$ at $\mathrm{RT}$, and finally resuspended in $50 \mu \mathrm{L} \mathrm{DMEM}+5 \% \mathrm{FBS}$. The cells were resuspended in $200 \mu \mathrm{L}$ DMEM $+5 \%$ FBS $\left(\sim 30 \times 10^{6}\right.$ cells $\left./ 100 \mu \mathrm{L}\right)$, mixed with the freshly prepared $50 \mu \mathrm{L} \mathrm{GNR}^{\mathrm{CD} 45}$, and incubated for $2 \mathrm{~h}$ at RT. Cells were washed twice with DMEM $+5 \%$ FBS by centrifuging at $340 \times g$ for $2 \mathrm{~min}$ at RT each time, and finally resuspended in $1 \mathrm{~mL}$ DMEM. A tube containing $200 \mu \mathrm{m}$ of DMEM $+5 \%$ FBS and $50 \mu \mathrm{L} \mathrm{GNR}^{\mathrm{CD} 45}$ was subjected to the same staining and washing conditions as the labeled cells. Using this tube as a control, the amount of free GNRs post wash was measured to be $<0.01 \mathrm{nM}$.

\subsection{Optical Coherence Tomography Imaging}

OCT images were acquired using a Ganymede ${ }^{\mathrm{TM}}$ HighResolution SD-OCT system (ThorLabs), equipped with a superluminescent diode (SLD) light source with a center wavelength of $900 \mathrm{~nm}$ and a $200 \mathrm{~nm}$ full operating bandwidth $(\Delta \lambda=800$ to $1000 \mathrm{~nm}$ ), which provided an axial $(Z)$ resolution of $2.1 \mu \mathrm{m}$ in water. The spectrometer acquires 2048 samples for each A-scan. The A-scan rate of the instrument was $30 \mathrm{kHz}$. All images were acquired using an LSM03 objective (ThorLabs), which provided
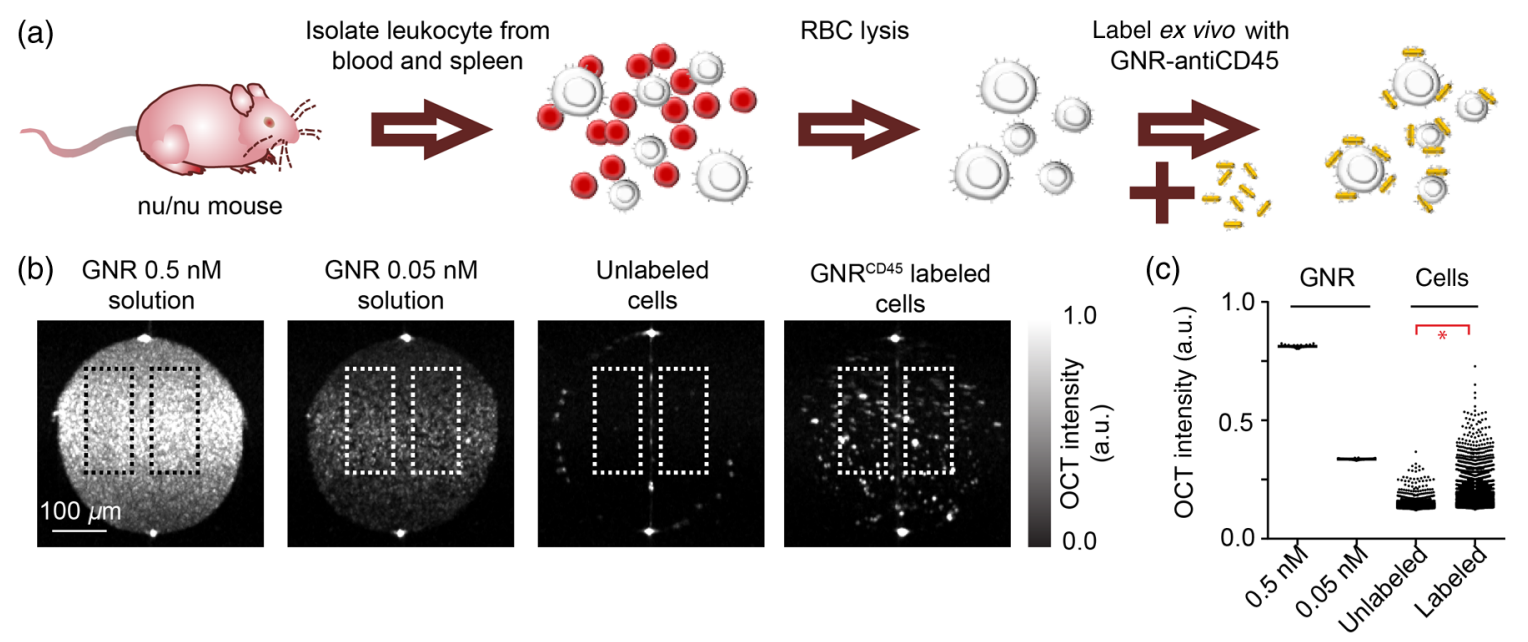

Fig. 1 Leukocyte labeling with GNR ${ }^{\mathrm{CD} 45}$. (a) Schematic of leukocyte labeling with $\mathrm{GNR}^{\mathrm{CD} 45}$. (b) From left to right OCT images of $0.5 \mathrm{nM}$ GNR solution, $0.05 \mathrm{nM}$ GNR solution, unlabeled leukocytes, and $\mathrm{GNR}^{\mathrm{CD} 45}$-labeled leukocytes. All samples were loaded into $400 \mu \mathrm{m}$ internal diameter glass capillaries for OCT imaging and imaged together. Scale bar $=100 \mu \mathrm{m}$. (c) Mean of log of OCT intensities from contiguous regions of size 10 pixels or larger within the dotted boxes shown in (b). Data pooled over $150 \mathrm{~B}$-scans from three independent experiments. ${ }^{*} p$ value $<0.001$ (unpaired $t$-test). 
a lateral $(X)$ resolution of $8 \mu \mathrm{m}(\mathrm{FWHM})$ and depth of field of $143 \mu \mathrm{m}$ in water. For two-dimensional (2-D) B-scans, images were acquired at $2 \mu \mathrm{m} /$ pixel in $X$ and $2 \mu \mathrm{m} /$ pixel in $Z$. One hundred B-scans were acquired for each 2-D dataset without averaging. For three-dimensional speckle variance scans, images were acquired at $400 \mathrm{~A}$-scans/B-scan at $5 \mu \mathrm{m} /$ pixel in $X, 2 \mu \mathrm{m} /$ pixel in $Z$, and 400 sets of B-scans in the $Y$ dimension at $5 \mu \mathrm{m} /$ pixel each. Eight B-scan averages were taken for each $X Z$ plane. The instrument is programmed to measure the spectrum of the SLD 25 times at the beginning of each acquisition. This measurement was used for normalizing images to the spectrum of the SLD. OCT intensities in tissues and in vessels were analyzed using MATLAB.

\subsection{Optical Coherence Tomography and Hyperspectral Imaging of Labeled Cells}

Single cell suspensions (labeled and unlabeled, see above), or standard GNR ${ }^{\mathrm{mPEG}}$ solutions $(0.05$ and $0.5 \mathrm{nM})$ were drawn into $400 \mu \mathrm{m}$ internal diameter glass capillaries and imaged with Ganymede at $2 \mu \mathrm{m} /$ pixel in $X$ and $2 \mu \mathrm{m} /$ pixel in $Z$ as described above. First, OCT intensities were calculated for individual pixels within regions inside the capillaries [Fig. 1(b)], which were devoid of reflection artifacts. Then, mean intensities were calculated from contiguous regions of 10 pixels or larger, which exhibited signal above background noise (measured from water-only capillaries). In addition, $\sim 20 \mu \mathrm{L}$ of cells from each sample was mounted on glass slides with CytoSeal 60 (electron microscopy sciences) for dark-field imaging. Darkfield hyperspectral microscopy was performed using a Cytoviva $^{\mathrm{TM}}$ system [Fig. 2(a)] equipped with a Fiber-Lite ${ }^{\mathrm{TM}}$ DC-950 light source, an Olympus UPlanFLN 100× $1.30 \mathrm{~N}$. A. oil objective, an Andor ${ }^{\mathrm{TM}} \mathrm{iXon} 3$ hyperspectral camera, and a Dage-MTI ${ }^{\mathrm{TM}}$ XLMCT digital color camera. Hyperspectral images were normalized to the light source spectrum of the Cytoviva instrument.

\subsection{Imaging of Live Mouse Retina Using Optical Coherence Tomography}

$\mathrm{Nu} /$ nu mice were anesthetized using $2.5 \%$ isofluorane $+\mathrm{O}_{2}(\mathrm{v} / \mathrm{v})$. Once adequately anesthetized, the mice were mounted onto a (a)

Hyperspectral imaging setup

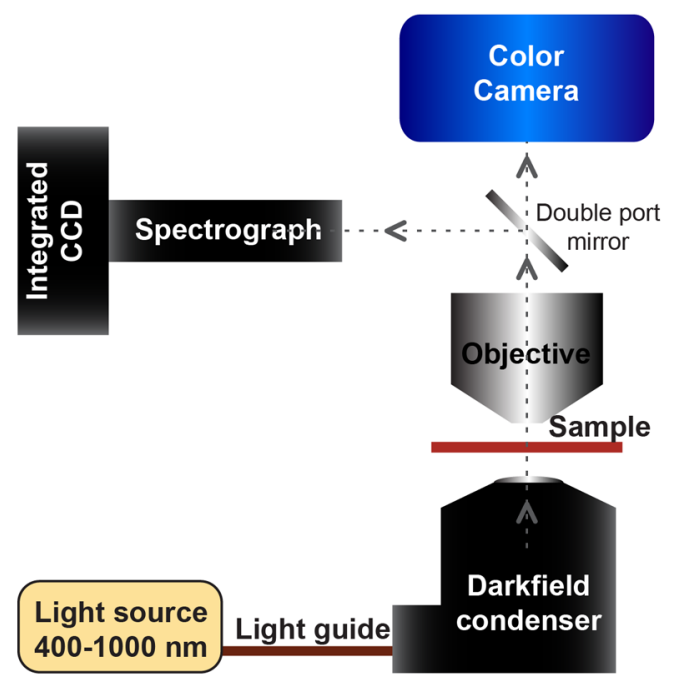

(b)

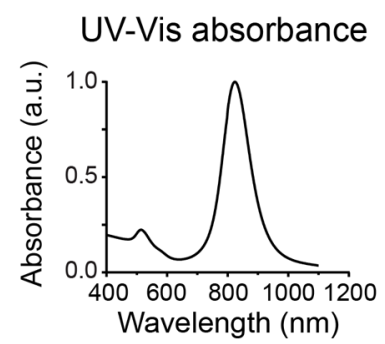

(c)

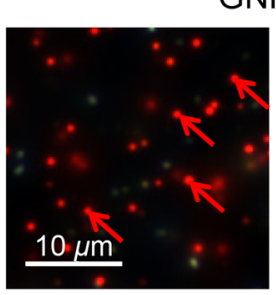

GNRs on slide

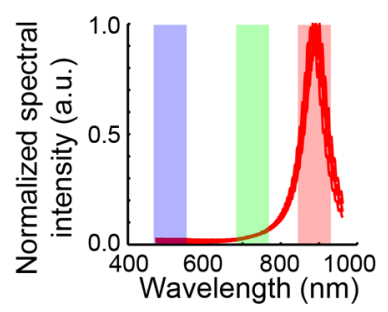

(d)

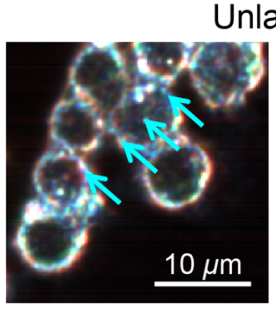

Unlabeled cells

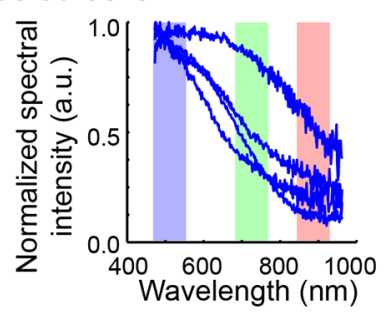

(e)
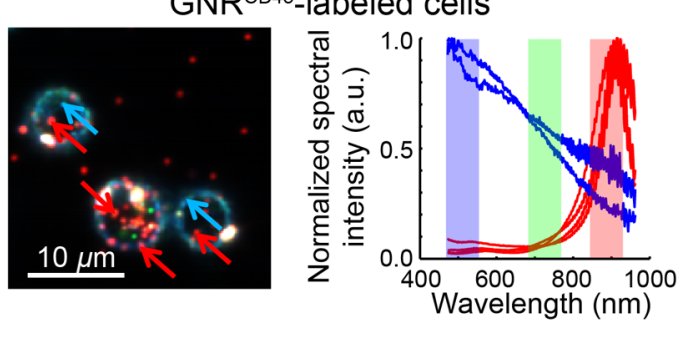

Fig. 2 Hyperspectral detection of GNRs in leukocytes labeled with GNR $^{\mathrm{CD} 45}$. (a) Diagrammatic representation of the Cytoviva ${ }^{\mathrm{TM}}$ hyperspectral imaging setup. Dotted line and arrows indicate the light path. (b) Absorbance of GNRs measured by UV-Vis. (c) Hyperspectral image of GNRs (peak $830 \mathrm{~nm}$ ) on a glass slide (left) and the entire spectrum (400 to $1000 \mathrm{~nm}$ ) of randomly chosen GNRs indicated by red arrows (right). (d) Hyperspectral image of unlabeled leukocytes, post RBC lysis, washed and overlain on a glass slide (left) and spectra of randomly chosen points indicated by blue arrows (right). (e) Leukocytes, after removal of RBCs by lysis, and labeled with GNR ${ }^{\mathrm{CD} 45}$ for $1 \mathrm{~h}$, washed, and overlain on a glass slide (left) and spectra of representative pixels indicated by red and blue arrows in their corresponding colors (right). All images were acquired with a $100 \times 1.3$ N.A. oil objective; (c) through (e) red, green, and blue highlights indicate bands that were used to represent the images on the left. Centers of bands are red $=887 \mathrm{~nm}$, green $=725 \mathrm{~nm}$, blue $=510 \mathrm{~nm}$; each band is Gaussian with an FWHM of $80 \mathrm{~nm}$. Scale bars $=10 \mu \mathrm{m}$. 
platform and secured with stereotactic devices (Fig. 3). With the mouse secured, the stage was tilted so that the mouse was on the side and the eye was facing up, with the top of the cornea being approximately parallel to the table. In this position, a plastic O-ring was carefully placed surrounding the eye using vacuum grease (Dow Corning), so as not to touch the cornea and to form a fluid-tight seal. Then, pupillary dilation was achieved by applying one drop each of $1 \%$ tropicamide (Bausch \& Lomb) and 2.5\% phenylephrine hydrochloride (Paragon BioTeck) to the eyes, for 2 min each. If any fluid leakage was observed after application of the dilating solutions, additional vacuum grease was applied toward the outer edge of the base of the O-ring. 2.5\% hypromellose solution (Gonak ${ }^{\mathrm{TM}}$, Akorn Inc.) was then placed over each eye as a contact solution. Enough hypromellose solution was applied to form an upward meniscus so that no air bubbles were formed when placing cover slips over the solution. Finally, 0.17-mm-thick glass coverslips were gently placed over the O-ring to complete a fluid-tight chamber over the dilated eye [Figs. 3(c) and 3(d)]. Anesthesia was continually maintained using a nose-cone delivering 1.5 to $2 \%$ isofluorane $+\mathrm{O}_{2}$. The level of anesthesia, hydration of the eye, and the body temperature of the mouse were continually monitored to prevent the formation of a cataract. Experiments where cataract formation was evident were not used for statistical analysis. OCT images of the live mouse retina were then acquired using Ganymede as described above.

\subsection{Optical Sensitivity of Contrast Enhancement in Live Retinae}

$\mathrm{Nu} / \mathrm{nu}$ mice were anesthetized and mounted for retina imaging as described earlier. Sensitivity assays were performed by injection of mPEG-conjugated GNRs $\left(\mathrm{GNR}^{\mathrm{PEG}}\right) .200 \mu \mathrm{L}$ of $10 \mathrm{nM}$ $\mathrm{GNR}^{\mathrm{mPEG}}$ was progressively injected intravenous (IV) via a tail vein catheter ( $29 \mathrm{G}$, Vevo) in steps of $25 \mu \mathrm{L}$. Imaging was performed after waiting for 3 min after each injection. Focus was established on the outer plexiform layer of the retina. B-scans and speckled variance images were acquired using Ganymede ${ }^{\mathrm{TM}}$, and image analysis was performed, as described earlier.

\subsection{Retinal Laser Injury Model}

$\mathrm{Nu} / \mathrm{nu}$ mice were anesthetized with intraperitoneal injections of $80 \mathrm{mg} / \mathrm{kg}$ ketamine (Vedco Inc.) and $10 \mathrm{mg} / \mathrm{kg}$ xylazine (Lloyd Inc.). Once adequately anesthetized, pupillary dilation was achieved as described above. Laser spots of $400 \mathrm{~mW}$ power, $100 \mu \mathrm{m}$ diameter for a pulse duration of $100 \mathrm{~ms}$ were performed to the retina using a Pascal system with a $525 \mathrm{~nm}$ laser.

\subsection{Dark-Field Histology of Leukocytes after Laser Injury in Mice Retina}

GNR labeled leukocytes were prepared (see above) and resuspended to $500 \mu \mathrm{L}$ at $20 \times 10^{6}$ cells $/ 100 \mu \mathrm{L}$. Mice retinae were then subjected to laser injury, and subsequently, a total of $100 \times 10^{6}$ labeled cells were injected IV via a tail vein catheter in $100 \mu \mathrm{L}$ steps $\left(20 \times 10^{6}\right.$ cells per injection). OCT imaging was performed after waiting for $3 \mathrm{~min}$ following every injection. There was an interval of $5 \mathrm{~min}$ between each $100 \mu \mathrm{L}$ injection. The mice were euthanized $4 \mathrm{~h}$ after injection, and eyes and spleens were harvested and saved in $10 \%$ formalin for histological analysis. Formalin-preserved organs were paraffin embedded and dissected into 5- $\mu$ m-thick sections. The sections were mounted without any staining on microscope slides using CytoSeal 60 mounting medium and cover glass. The spleen was used as a positive control since labeled leukocytes are expected to home into the spleen regardless of injury or inflammation. Dark-field hyperspectral microscopy was (a)

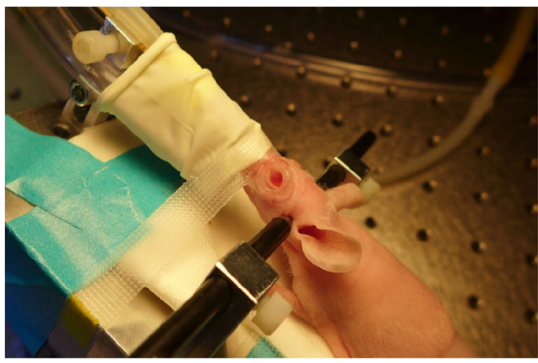

(c)

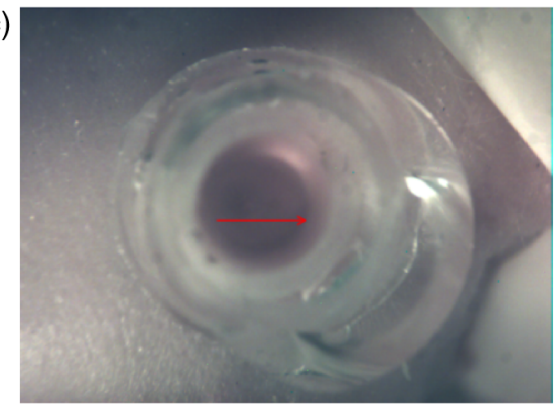

(b)

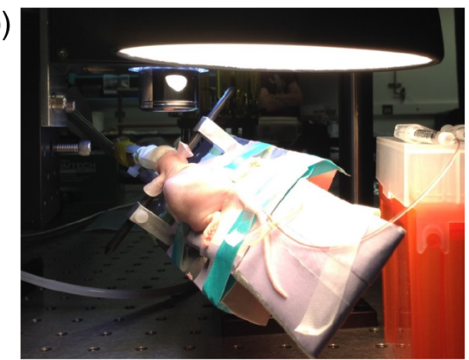

(d)

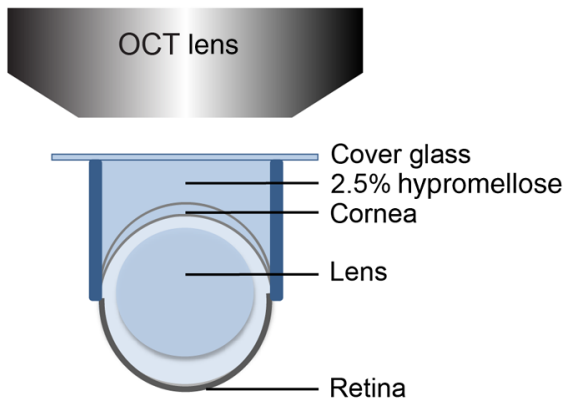

Fig. 3 Setup for imaging of live mouse retina. (a) Inclined stage with an anesthetized nu/nu mouse restrained for retinal imaging. (b) Mouse is kept warm using a lamp, anesthesia is maintained by 1.5 to $2 \%$ isoflurane in $\mathrm{O}_{2}(\mathrm{v} / \mathrm{v})$ delivered by a nose cone, and a tail vein catheter is inserted and secured for intravenous delivery of GNRs or cells. (c) Photograph and (d) diagram of a mouse eye encased using $2.5 \%$ hypromellose solution in a fluid-tight chamber. 
performed using the Cytoviva ${ }^{\mathrm{TM}}$ system described above, with an Olympus UPlanFLN 40×, 0.75 N.A. air objective. Hyperspectral images normalized to the light source spectrum of the Cytoviva instrument were saved. In the hyperspectral images, GNRs are expected to exhibit peaks at $\sim 887 \mathrm{~nm}$ spectral region.

\subsection{Use of Animals}

All experiments were performed in strict accordance within the rules and guidelines set by the Institutional Animal Care and Use Committee and the Animal Studies Committee's Guidelines for the Care and Use of Research Animals (APLAC 27602) at Stanford University.

\section{Results}

\subsection{Optical Coherence Tomography and Hyperspectral Imaging of GNR ${ }^{\mathrm{CD} 45}$-Labeled Leukocytes}

We leveraged the enhanced scattering property of our custom synthesized GNRs to increase the OCT contrast of leukocytes. We first labeled freshly isolated leukocytes with GNR ${ }^{\mathrm{CD} 45}$ [Fig. 1(a)] and then imaged the $\mathrm{GNR}^{\mathrm{CD} 45}$-labeled leukocytes by OCT. GNR ${ }^{\mathrm{CD} 45}$-labeled leukocytes were significantly brighter than unlabeled cells in terms of absolute scattering intensity [Figs. 1(b) and 1(c)]. At least 6\% of labeled cells had absolute intensity above a $0.05 \mathrm{nM}$ GNR solution. To verify leukocyte labeling by GNRs, we imaged these cells as well as a GNRs-only sample and an unlabeled cell sample with hyperspectral dark-field microscopy. The GNRs-only sample exhibited spectra with characteristic peaks at or about $887 \mathrm{~nm}$ with an FWHM of $\sim 100 \mathrm{~nm}$ [Fig. 2(c)]. GNR absorbance measured by UV-Vis exhibited a similar FWHM [Fig. 2(b)]. The unlabeled cells exhibited ambient scattering encompassing a broad spectral region [Fig. 2(d)]. GNR-labeled cells had distinct GNRs both on the surface, as well as inside the cell [Fig. 2(e)]. The GNRs associated with the cells exhibited the characteristic spectra that were observed for GNRs alone. Within the labeled cells, regions that did not contain GNRs had ambient scattering spectra similar to unlabeled cells. These results indicate leukocytes can be efficiently labeled with $\mathrm{GNR}^{\mathrm{CD} 45}$, which can alter scattering and spectral properties of these leukocytes to allow detection by OCT imaging.

\subsection{Optical Coherence Tomography Imaging of Living Mouse Retinae by Gold Nanorod Enhanced Detection Sensitivity}

We next investigated the detection sensitivity of GNRs in vivo in mice retinae to deduce the degree of labeling necessary to allow detection of labeled cells in retinae. To adapt the Ganymede ${ }^{\mathrm{TM}}$ system for retinal imaging, we encased the eyes of anesthetized mice in a fluid-tight chamber forming a plano-convex lens system allowing the focal length of the Ganymede system to reach all the retinal layers (Fig. 3). Next, we progressively injected $200 \mu \mathrm{L}$ total of a $10 \mathrm{nM}$ solution $\mathrm{GNR}^{\mathrm{mPEG}}$ and measured OCT intensities inside retinal blood vessels [Fig. 4(a)]. OCT intensity increased within the retinal blood vessels following injection of GNR ${ }^{\mathrm{mPEG}}$ [Fig. 4(b)]. Changes in OCT intensities in retinal blood vessels could be distinctly differentiated from baseline intensities starting at an effective GNR concentration of $0.5 \mathrm{nM}$ in blood [Fig. 4(c)]. At higher GNR concentrations in the blood, blood vessels with diameter $<20 \mu \mathrm{m}$ could also be detected. GNR ${ }^{\mathrm{mPEG}}$ persisted in blood circulation for $\sim 4 \mathrm{~h}$ until they were cleared out, which was denoted by a return to preinjection scattering intensity levels [Fig. 4(d)]. It needs to be mentioned here that after GNR injection, when focus was established in the outer plexiform layer, we noticed lower intensities of backscattered light from the choroidal vasculature compared to the vessels within the plexiform layers. To confirm the presence of GNRs in the retinal blood vessels, we imaged unstained $5-\mu \mathrm{m}$-thick formalin-fixed paraffin embedded sections (without removing the paraffin) of retinae of mice injected with $200 \mu \mathrm{L}$ of $10 \mathrm{nM} \mathrm{GNR}{ }^{\text {mPEG }}$ and euthanized $1 \mathrm{~h}$ postinjection [Fig. 4(e)]. Characteristic spectra of GNRs (887 nm, represented in red) were clearly visible inside the retinal vessels, confirming the presence of stable GNR ${ }^{\text {mPEG }}$ within these vessels. These results show that using our OCT imaging technique, GNRs can be detected at a sensitivity of $\sim 0.5 \mathrm{nM}$ inside retinal blood vessels following IV injection.

\subsection{Migration of $\mathrm{GNR}^{\mathrm{CD} 45}$-Labeled Leukocytes to Sites of Laser-Induced Retinal Injury}

We expected labeled leukocytes with intensities greater than a $0.5 \mathrm{nM}$ equivalent of GNRs to be detected in the retina by contrast-enhanced OCT, based on our GNR sensitivity measurements. We next imaged sites of laser-induced injury in nu/nu mice retinae to detect leukocyte migration within these areas. Following laser injury to the retina, we expect an increase in infiltrating leukocytes (predominantly neutrophils) to the site of injury. ${ }^{21}$ To this end, we injected $\mathrm{GNR}^{\mathrm{CD} 45}$-labeled leukocytes in mice $30 \mathrm{~min}$ after laser injury. We were able to distinguish a few GNR ${ }^{\mathrm{CD} 45}$-labeled leukocytes inside retinal blood vessels using enhanced OCT contrast [Fig. 5(a) and Video 1]. We measured the speed of the observed moving cells within the smaller capillaries to be $\sim 90 \mu \mathrm{m} / \mathrm{s}$. These increased signals were more evident in outer plexiform layer, which exhibited low OCT intensity prior to injection of cells. As unlabeled leukocytes were poorly scattering (Fig. 1) and the amount of free GNR was at least 50-fold lower than OCT detection limits in vivo, these signals were unlikely from free GNRs or unlabeled leukocytes. Additionally, there was a small but significant increase in OCT intensity inside larger vessels after $60 \times 10^{6}$ and $100 \times 10^{6}$ GNR-labeled cells had been injected [Fig. 5(b)] These results are indicative of the change in blood vessel intensity due to injected GNR-labeled cells. However, we were unable to distinguish $\mathrm{GNR}^{\mathrm{CD} 45}$-labeled cells at the sites of injury, mainly because of speckle noise within the injured tissue that had intensities greater than our previously calculated detection limit of $0.5 \mathrm{nM}$ GNR equivalent. Thus, we next investigated the presence of GNR-labeled cells in the retinae at the sites of injury by histological analysis using hyperspectral microscopy [Fig. 6(a)]. Two hours after laser injury and injection of GNRlabeled leukocytes, we observed characteristic GNR peaks (887 $\mathrm{nm}$, represented in red) near the site of laser injury in mice retinae. These GNRs at the site of injury were closely associated with cells embedded in the choroidal region. GNR spectra were absent in the corresponding nonlasered eye obtained from the same mice [Fig. 6(b)]. We expected labeled leukocytes to constitutively migrate to the spleen. Thus, to further confirm the presence of injected GNR-labeled cells in these mice, we imaged histological spleen sections of the same mice and observed a large number of $\mathrm{GNR}^{\mathrm{CD} 45}$-labeled cells inside the spleens [Fig. 6(b)]. We also imaged sections from eyes of 
(a)

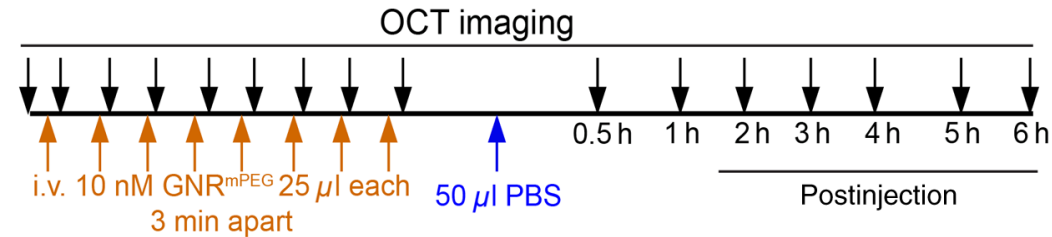

(b) 0

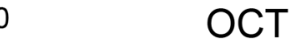

$1 \quad(c)$
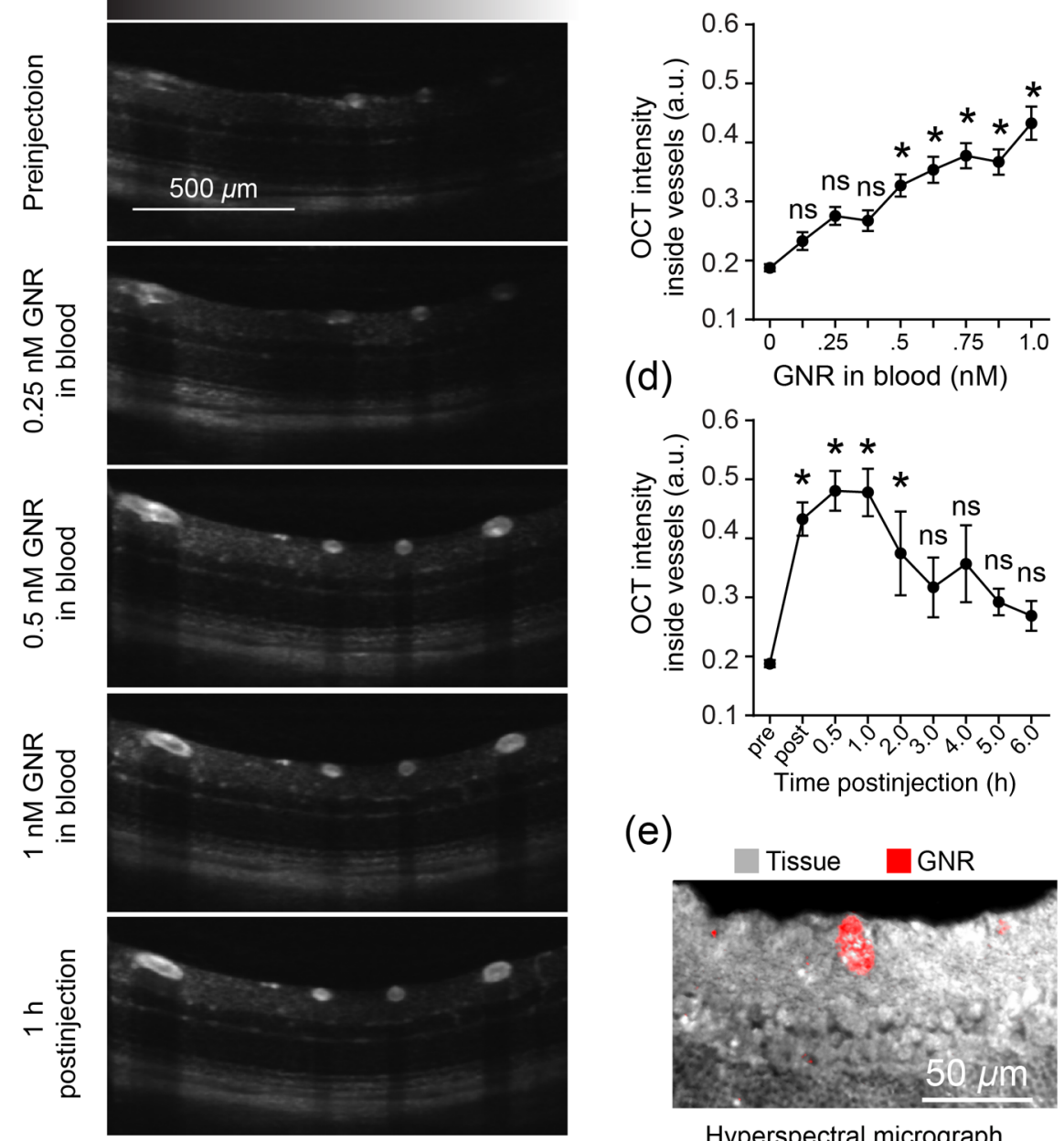

(e)

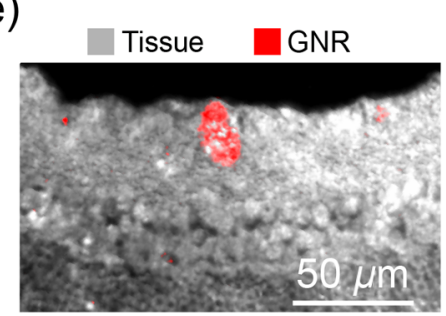

Hyperspectral micrograph

Fig. 4 Contrast-enhanced OCT imaging of retinal vessels after intravenous GNR $^{\mathrm{mPEG}}$ injection. (a) Experimental design. Orange arrows indicate the time points for IV injection of GNR ${ }^{m P E G}$. Black arrows indicate the times at which OCT imaging was performed. (b) Representative OCT images of mice retinae before and after GNR ${ }^{\mathrm{mPEG}}$ injection. Conditions corresponding to the images are indicated on the left. Scale bar $=500 \mu \mathrm{m}$. (c) and (d) Measure of OCT intensity inside retinal blood vessels after GNR $^{m P E G}$ injection. (c) Sensitivity measurements and (d) measure of GNR ${ }^{m P E G}$ signal decay from blood postinjection. Mean \pm SEM; * $p$ value $<0.05$, ns $=$ not significant; all conditions in (c) and (d) are compared with preinjection images using one-way analysis of variance. Data represent three independent experiments. (e) Hyperspectral image of unstained 5- $\mu \mathrm{m}$-thick formalin-fixed paraffin embedded section of a mouse retina $1 \mathrm{~h}$ after IV injection of GNR ${ }^{\mathrm{mPEG}}$. Gray-ambient scattering from tissue, red-pixels with characteristic spectra from GNRs. Scale bar $=50 \mu \mathrm{m}$. Note the presence of GNRs inside a large vessel at the center.

mice that received laser thermotherapy but were not injected with GNR-labeled cells [Fig. 6(c)]. Expectedly, characteristic GNR spectra were absent in these control samples. From these results, we conclude that the hyperspectral GNR signals observed in the retina near sites of laser injury, but not near noninjured sites, are most likely due to $\mathrm{GNR}^{\mathrm{CD} 45}$-labeled cells migrating preferentially to the sites of laser-induced injury in mice retinae and that these cells can be detected using hyperspectral histological analysis. While our current implementation of OCT can detect these cells inside vessels, they were not observable in the injured region due to excess speckle noise.

\section{Discussion}

In this report, we have demonstrated that contrast agents such as GNRs and the imaging depth and resolution achievable with 
(a)
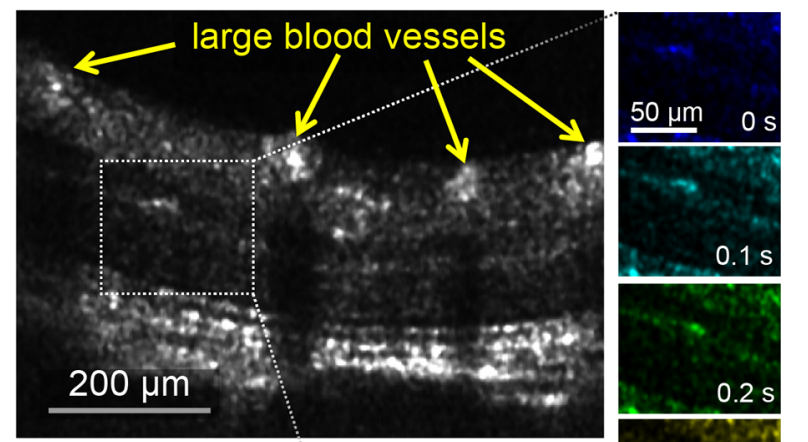

(b)
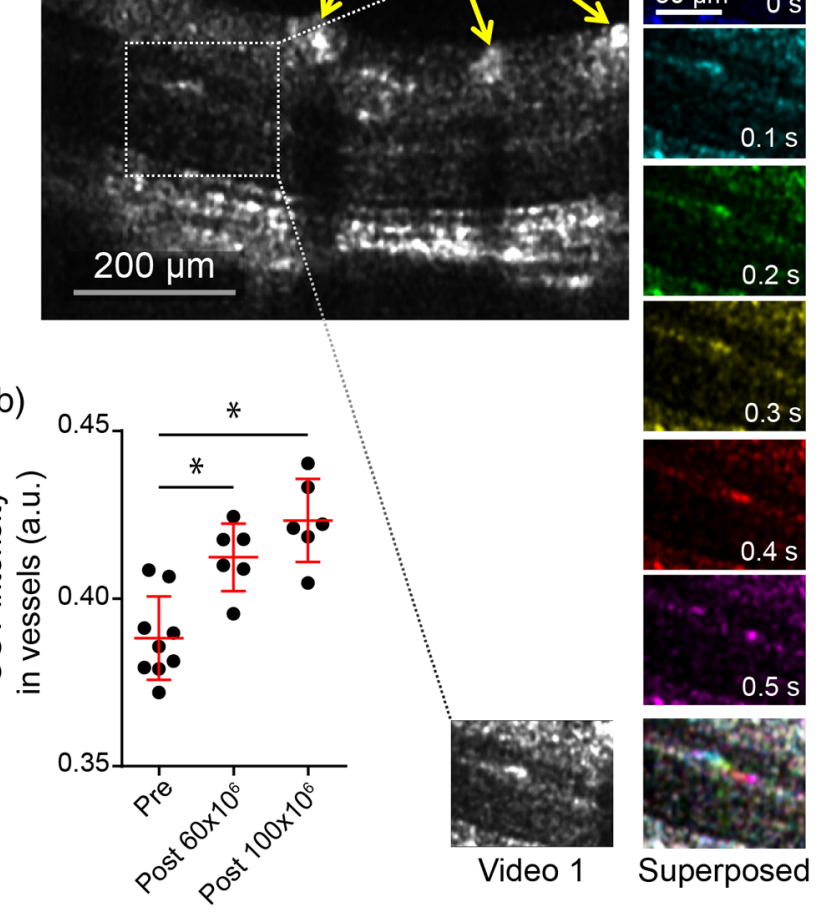

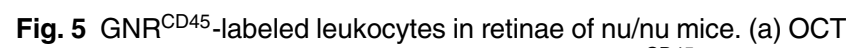
image of a nu/nu mouse retina postinjection of $\mathrm{GNR}^{\mathrm{CD} 45}$-labeled leukocytes (left, large blood vessels indicated by yellow arrows), and six sequential images of the boxed area inside the OCT image (each average of three B-scans) color-coded by time. Elapsed time is indicated inside each frame, with the first frame (blue) assigned time point 0 . Superposition of the six frames (right) shows a multicolored path, indicative of a moving cell within a capillary. Scale bar $=200 \mu \mathrm{m}$ (left) and $50 \mu \mathrm{m}$ (right). (also refer to Video 1, MOV, $5.91 \mathrm{MB}$ ) [URL: http:// dx.doi.org/10.1117/1.JBO.21.6.066002.1]. (b) OCT intensity in blood vessels shown in (a), with each data point representing intensity inside a single blood vessel summed over 100 B-scans before injection (pre) and after injection of $60 \times 10^{6}$ and $100 \times 10^{6}$ total GNR $^{\text {CD45 }}$-labeled leukocytes. Red line and bars indicate Mean \pm SD. ${ }^{*} p$ value $<0.05$ (unpaired $t$-test).

OCT can provide an imaging technology capable of detecting signals at single cell level in a living mouse retina. This minimally invasive approach can achieve imaging depths of several millimeters and axial resolution of up to $2.1 \mu \mathrm{m}$, thus providing superior detection capabilities than current noninvasive fluorescence imaging techniques. Moreover, the measured detection sensitivity for GNRs $(0.5 \mathrm{nM})$ makes this OCT-based approach particularly attractive. Our custom synthesized GNRs ${ }^{15}$ provide better depth and resolution in OCT compared to smaller GNRs used in previous studies to enhance contrast in tissues and in vivo. ${ }^{8,12,13}$ We further demonstrated that leukocytes obtained from mouse blood and spleen tissue can be successfully labeled with scattering contrast agents such as GNRs via a targeted labeling strategy. Such GNR-labeling can be utilized to enhance scattering intensity of these otherwise poorly scattering cells, allowing them to be detected by OCT. This, to our knowledge, is the first demonstration of targeted cell labeling using contrast agents that enhance scattering. This work demonstrates that GNRs can be used as multifaceted probes for contrast-enhanced OCT imaging and dark-field hyperspectral microscopy due to their unique scattering spectra. The ability to observe these nanoparticles in hyperspectral microscopy can corroborate the spectral images observed with OCT.

Migrating GNR-labeled leuokocytes were detected inside retinal blood vessels but were undetected at the site of tissue injury due to excess speckle noise. Improvements in the GNR labeling technique and utilization of instrumentation to reduce speckled noise may improve our OCT system to enable the detection of GNR-labeled objects within extravascular tissue. Currently, backscattered intensity after GNR injection in the choroid is comparable to preinjection speckle noise in the choroid, limiting the systems capability to detect GNR-labeled cells in the choroid. With our current imaging system, algorithmic removal of tissue speckle noise is insufficient to obtain the signal-to-noise ratio necessary for identification of GNR-labeled cells outside the blood vessels. In future studies, the ability of our GNRs to scatter optimally within specific wavelengths of incident light may be utilized to differentiate GNR spectra from ambient scattering from the retinal layers. After GNR injection, we also observed noticeably low signal intensity at the center of larger vessels, compared to the edges. This may be attributed to fringe washout commonly observed in large vessels in spectral domain $\mathrm{OCT}^{22}$ In future studies, improved instrumentation and computational techniques can be used to remove washout artifacts.

We needed to make some adjustments for detecting GNRs in the retina by hyperspectral microscopy. First, we observed a shift in the GNR spectral peak ( $\sim 830 \mathrm{~nm}$ in UV-Vis), which may be attributed to changes in plasmonic property of the GNRs due to high refractive index mounting media ${ }^{23}$ and adsorbed proteins on their surface. ${ }^{24}$ Even though H\&E staining of histological section provides excellent anatomical references for the retinal layers, such staining interfered with the spectral detection of GNRs, leading us to image unstained sections. Additionally, to prevent loss of unbound GNR $^{\mathrm{mPEG}}$ inside blood vessels, we imaged these sections without deparaffinization.

Our OCT imaging system can be extended in the future to image the spatiotemporal behavior of immune cells inside tissues and to detect microstructures or biomarkers within such tissues by specific labeling using functionalized GNRs. We envision that the methods described herein may be used in the future to shed light on the roles of immune cells ${ }^{25-28}$ and biomarkers, such as amyloid beta, ${ }^{29}$ VEGFR $1,{ }^{30}$ and $\alpha_{\mathrm{v}} \beta_{3}{ }^{31}$ in atrophic age-related macular degeneration and other retinal disorders. These results will help us better understand the cellular and molecular basis of disease progression via longitudinal studies. OCT has been recently used in conjunction with imaging techniques such as scanning laser ophthalmoscopy, ${ }^{32-34}$ fundus autofluorescence, ${ }^{35}$ fluorescein angiography, ${ }^{32,36,37}$ ultrasound ${ }^{38}$ and Indocyanine Green angiography,${ }^{39}$ helping in better diagnosis of diseased tissue. Multiphoton imaging has also been used to study fluorescent cells in the mouse retina. ${ }^{40}$ While such fluorescence imaging can provide subcellular resolution, these techniques are limited by depth of penetration and the use of laser powers above the safety limits for humans. ${ }^{41}$ Thus, our strategies for leveraging the benefits of depth and resolution offered by OCT, as well as the enhanced scattering by functionalized GNRs may complement these existing clinical imaging techniques and help in better diagnosis and personalized treatments of disorders. 
(a)

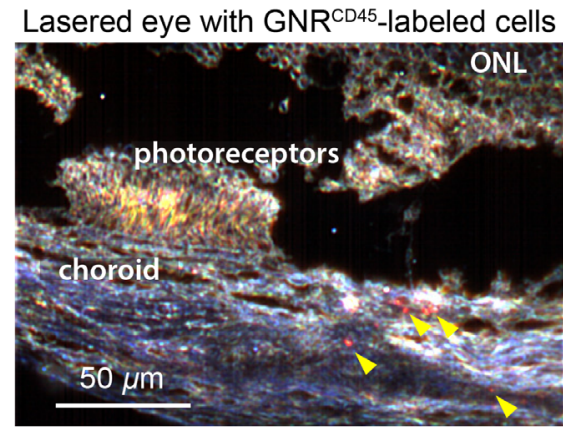

(c)

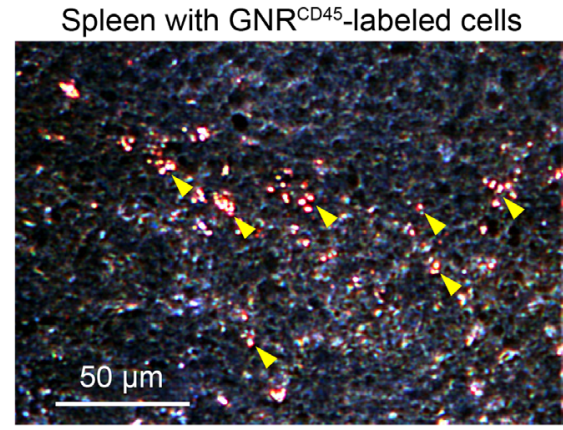

(b)

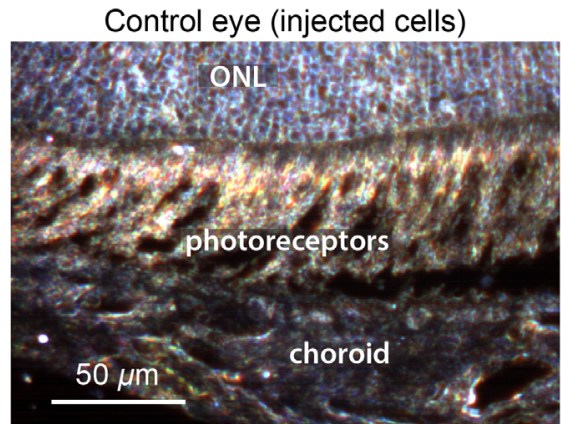

(d)

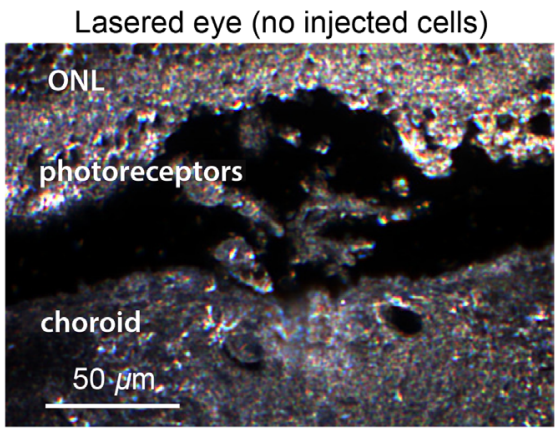

\section{$\mathrm{R}=887 \pm 80 \mathrm{~nm} \mathrm{G}=725 \pm 80 \mathrm{~nm} \mathrm{~B}=510 \pm 80 \mathrm{~nm}$}

Fig. 6 Hyperspectral images of GNR-labeled leukocytes inside lasered eyes of mice. (a) Hyperspectral image of 5- $\mu \mathrm{m}$-thick unstained section of lasered eye and (b) the corresponding nonlasered control eye of $\mathrm{nu} / \mathrm{nu}$ mice that received GNR ${ }^{\mathrm{CD} 45}$-labeled cells. The choroid, photoreceptor, and outer nuclear layer $(\mathrm{ONL})$ regions of the retina have been marked inside the image. Red GNR-labeled cells are indicated by yellow arrowheads. (c) Hyperspectral image of $5-\mu \mathrm{m}$-thick unstained section of spleen of mice injected with GNR-labeled cells. Red GNR-labeled cells indicated by yellow arrowheads. (d) Hyperspectral image of $5-\mu \mathrm{m}$-thick unstained section of laser-treated eye of control mice without injected GNR-labeled cells. Center of bands are red $=887 \mathrm{~nm}$, green $=725 \mathrm{~nm}$, blue $=510 \mathrm{~nm}$; each band is Gaussian with an FWHM of $80 \mathrm{~nm}$. Scale bars $=50 \mu \mathrm{m}$

\section{Acknowledgments}

We thank Bryan Knysh, Taylor Stilwell, Yong Tang, Noralyn Guzman, Daniel V. Palanker, Yonatan Winetraub, and Byron Cheatham for technical assistance and discussions. This research was supported by grants from the Arnold and Mabel Beckman Initiative for Macular Research (Grant \#1407), the Claire Giannini Fund, the United States Air Force (FA955015-1-0007), Stanford Bio-X Interdisciplinary Initiative Program (IIP6-43), National Institutes of Health (NIH DP50D012179), the National Science Foundation (NSF CBET1434465), the Donald E. and Delia B. Baxter Foundation, and the Skippy Frank Foundation. Adam de la Zerda is a Pew-Stewart Scholar for Cancer Research supported by the Pew Charitable Trusts and the Alexander and Margaret Stewart Trust. In addition to these sources, we would like to thank the Stanford Nanocharacterization Lab for a Nano Seed Grant for GNR characterization. Orly Liba is grateful for a Stanford Bowes Bio-X Graduate Fellowship. Elliott D. SoRelle wishes to acknowledge funding from the Stanford Biophysics Program training grant (T32 GM-08294).

\section{References}

1. C. A. Puliafito et al., "Imaging of macular diseases with optical coherence tomography," Ophthalmology 102(2), 217-229 (1995).

2. M. R. Hee et al., "Optical coherence tomography of age-related macular degeneration and choroidal neovascularization," Ophthalmology 103(8), 1260-1270 (1996).
3. A. de la Zerda et al., "Ultrahigh sensitivity carbon nanotube agents for photoacoustic molecular imaging in living mice," Nano Lett. 10(6), 2168-2172 (2010).

4. A. de la Zerda et al., "Photoacoustic ocular imaging," Opt. Lett. 35(3), 270-272 (2010).

5. A. de la Zerda et al., "Advanced contrast nanoagents for photoacoustic molecular imaging, cytometry, blood test and photothermal theranostics," Contrast Media Mol. Imaging 6(5), 346-369 (2011).

6. M. F. Kircher et al., "A brain tumor molecular imaging strategy using a new triple-modality MRI-photoacoustic-Raman nanoparticle," Nat. Med. 18(5), 829-834 (2012).

7. T. S. Troutman, J. K. Barton, and M. Romanowski, "Optical coherence tomography with plasmon resonant nanorods of gold," Opt. Lett. 32(11), 1438-1440 (2007).

8. B. Wang et al., "Gold nanorods as a contrast agent for Doppler optical coherence tomography," PLoS One 9(3), e90690 (2014).

9. R. K. Chhetri et al., "Probing biological nanotopology via diffusion of weakly constrained plasmonic nanorods with optical coherence tomography," Proc. Natl. Acad. Sci. 111(41), E4289-E4297 (2014).

10. A. L. Oldenburg et al., "Motility-, autocorrelation-, and polarizationsensitive optical coherence tomography discriminates cells and gold nanorods within 3-D tissue cultures," Opt. Lett. 38(15), 2923-2926 (2013).

11. Y. Jia et al., "Spectral fractionation detection of gold nanorod contrast agents using optical coherence tomography," Opt. Express 23(4), 42124225 (2015).

12. M. Gabriele Sandrian et al., "Inflammatory response to intravitreal injection of gold nanorods," Br. J. Ophthalmol. 96(12), 1522-1529 (2012).

13. A. de la Zerda et al., "Optical coherence contrast imaging using gold nanorods in living mice eyes," Clin. Exp. Ophthalmol. 43(4), 358-366 (2014). 
14. J. M. Tucker-Schwartz et al., "In vivo imaging of nanoparticle delivery and tumor microvasculature with multimodal optical coherence tomography," Biomed. Opt. Express 5(6), 1731-1743 (2014).

15. E. D. SoRelle et al., "Biofunctionalization of large gold nanorods realizes ultrahigh-sensitivity optical imaging agents," Langmuir 31(45), 12339-12347 (2015).

16. J. S. Schuman et al., "Optical coherence tomography: a new tool for glaucoma diagnosis," Curr. Opin. Ophthalmol. 6(2), 89-95 (1995).

17. N. Khlebtsov and L. Dykman, "Biodistribution and toxicity of engineered gold nanoparticles: a review of in vitro and in vivo studies," Chem. Soc. Rev. 40(3), 1647-1671 (2011).

18. S. K. Libutti et al., "Phase I and pharmacokinetic studies of CYT-6091, a novel PEGylated colloidal gold-rhTNF nanomedicine," Clin. Cancer Res. 16(24), 6139-6149 (2010).

19. J. M. Stern et al., "Initial evaluation of the safety of nanoshell-directed photothermal therapy in the treatment of prostate disease," Int. J. Toxicol. 35(1), 38-46 (2015).

20. L. Cheng et al., "Functional nanomaterials for phototherapies of cancer," Chem. Rev. 114(21), 10869-10939 (2014).

21. J. Zhou et al., "Neutrophils promote experimental choroidal neovascularization," Mol. Vis. 11, 414-424 (2005).

22. S. H. Yun et al., "Motion artifacts in optical coherence tomography with frequency-domain ranging," Opt. Express 12(13), 2977-2998 (2004).

23. T. Okamoto, I. Yamaguchi, and T. Kobayashi, "Local plasmon sensor with gold colloid monolayers deposited upon glass substrates," Opt. Lett. 25(6), 372-374 (2000).

24. A. L. Chen et al., "Quantifying spectral changes experienced by plasmonic nanoparticles in a cellular environment to inform biomedical nanoparticle design," Nanoscale Res. Lett. 9(1), 454 (2014).

25. F. Sennlaub et al., "CCR2(+) monocytes infiltrate atrophic lesions in age-related macular disease and mediate photoreceptor degeneration in experimental subretinal inflammation in Cx3crl deficient mice," EMBO Mol. Med. 5(11), 1775-1793 (2013).

26. J. G. Hollyfield et al., "Oxidative damage-induced inflammation initiates age-related macular degeneration," Nat. Med. 14(2), 194-198 (2008).

27. V. L. Perez et al., "The eye: a window to the soul of the immune system," J. Autoimmun. 45, 7-14 (2013).

28. N. Patel et al., "Circulating anti-retinal antibodies as immune markers in age-related macular degeneration," Immunology 115(3), 422-430 (2005).

29. J. Hoh Kam, E. Lenassi, and G. Jeffery, "Viewing ageing eyes: diverse sites of amyloid beta accumulation in the ageing mouse retina and the up-regulation of macrophages," PLoS One 5(10), e13127 (2010).

30. H. Huang et al., "VEGF receptor blockade markedly reduces retinal microglia/macrophage infiltration into laser-induced CNV," PLoS One 8(8), e71808 (2013).

31. J. Beutel, M. Rudolf, and S. Grisanti, "Current and future therapies for age-related macular degeneration," Expert Opin. Emerging Drugs 14(2), 341-362 (2009).

32. S. McLenachan et al., "Angiography reveals novel features of the retinal vasculature in healthy and diabetic mice," Exp. Eye Res. 138, 6-21 (2015).

33. R. J. Zawadzki et al., "Adaptive-optics SLO imaging combined with widefield OCT and SLO enables precise 3D localization of fluorescent cells in the mouse retina," Biomed. Opt. Express 6(6), 2191-2210 (2015).

34. P. F. Sharp et al., "The scanning laser ophthalmoscope-a review of its role in bioscience and medicine," Phys. Med. Biol. 49(7), 1085-1096 (2004).

35. P. C. Lim and C. J. Layton, "Prognostic implications of imaging in atrophic macular degeneration and its use in clinical practice and clinical trial design," Clin. Exp. Ophthalmol. (2015).

36. S. W. Kang, C. Y. Park, and D. I. Ham, "The correlation between fluorescein angiographic and optical coherence tomographic features in clinically significant diabetic macular edema," Am. J. Ophthalmol. 137(2), 313-322 (2004).

37. T. Otani and S. Kishi, "Correlation between optical coherence tomography and fluorescein angiography findings in diabetic macular edema," Ophthalmology 114(1), 104-107 (2007).

38. S. J. Kang et al., "Optical coherence tomographic analysis of in-stent neoatherosclerosis after drug-eluting stent implantation," Circulation 123(25), 2954-2963 (2011).

39. A. G. Podoleanu et al., "Investigations of the eye fundus using a simultaneous optical coherence tomography/indocyanine green fluorescence imaging system," J. Biomed. Opt. 12(1), 014019 (2007).
40. G. Palczewska et al., "Noninvasive two-photon microscopy imaging of mouse retina and retinal pigment epithelium through the pupil of the eye," Nat. Med. 20(7), 785-789 (2014).

41. P. Stremplewski et al., "Periscope for noninvasive two-photon imaging of murine retina in vivo," Biomed. Opt. Express 6(9), 3352-3361 (2015).

Debasish Sen is a scientist at Asterias Biotherapeutics based in Fremont, CA, USA. He received his $\mathrm{PhD}$ in biological sciences from the UC Irvine and was a postdoctoral staff research associate in the de la Zerda lab, Structural Biology, Stanford University. His research interests include studying the effects of single immune cell dynamics on the overall immune response and using novel imaging technologies to improve medical diagnosis and better understand the immunopathology of diseases.

Elliott $\mathrm{D}$. SoRelle is a biophysics $\mathrm{PhD}$ candidate conducting research in the de la Zerda Lab at Stanford University. He received his BSc in biochemistry and cell biology and his BA in visual arts from Rice University, where he graduated magna cum laude. His interests include biophotonics and nanoparticles for contrast-enhanced imaging. He has taught courses on the biology and chemistry of whisky production and currently teaches immunology for Biochips, Imaging and Nanomedicine at Stanford University.

Orly Liba is a $\mathrm{PhD}$ candidate in electrical engineering and a researcher in the de la Zerda lab, Structural Biology, Stanford University. She has received a BSc in electrical engineering, a BA in physics from the Technion, Israel, and an MSc in electrical engineering from Tel-Aviv University. Before Stanford, she worked as an algorithms engineer and team leader in image processing at two startups. She is currently interested in developing imaging technologies to better assist medical diagnosis.

Roopa Dalal is a life science research professional with the Department of Ophthalmology at Stanford University. Her research interests include histological effects of lasers, retina prosthetics, and immunochemical effects of drugs to cure AMD and blindness. She received her BSc and MSc from University of Mumbai, India, and an MS from Illinois State University.

Yannis M. Paulus is a surgical retina specialist and assistant professor (tenure track) at the University of Michigan Department of Ophthalmology and Visual Sciences and Department of Biomedical Engineering. He has a particular interest in developing novel retinal imaging systems and therapeutic techniques, including lasers, surgical techniques, and molecular imaging. He has received several entrepreneurship awards and grants, published more than 50 peerreviewed publications, and serves on the editorial board of 5 major publications.

Tae-Wan Kim is a clinical associate professor in the Department of Ophthalmology of Seoul National University, College of Medicine. He holds a PhD degree from the College of Medicine at Seoul National University. In over 10 years, he has accomplished important tasks in the following areas: age-related macular degeneration, diabetic retinopathy, retinal detachment, hereditary retinal disorders, and endogenous autoimmune uveitis. He also seeks to conduct some projects for molecular imaging of retinal disorders.

Darius M. Moshfeghi is professor of ophthalmology at Stanford University School of Medicine and is director of pediatric vitreoretinal surgery, as well as director of ophthalmic telemedicine. He is the founder of the Stanford University Network for Diagnosis of Retinopathy of Prematurity (SUNDROP) and leads the Newborn Eye Screen Testing program and Global Universal Eye Screen Testing programs at Stanford University, which seek to explore the benefits of universal eye screening of normal term infants.

Adam de la Zerda is an assistant professor in structural biology and electrical engineering at Stanford University. He received his BSc in computer engineering from Technion Israel Institute of Technology, and $\mathrm{MSc}$ and $\mathrm{PhD}$ in electrical engineering from Stanford University. He received numerous awards, including Forbes 30under-30 and NIH Director's Early Independence Award. He is developing imaging technologies that can shed light on the spatial-temporal behaviors of biomolecules in living subjects. 